\title{
STUDIES ON THE GENERALIZED SHWARTZMAN REACTION*
}

\author{
I. General Observations Concerning the Phenomenon \\ By LEWIS THOMAS, M.D., AND ROBERT A. GOOD, $\ddagger$ M.D. \\ (From the Heart Hospital Research Laboratories and the Department of \\ Pediatrics, University of Minnesota Medical School, Minneapolis)
}

Plate 29

(Received for publication, July 30, 1952)

Following the description by Shwartzman (1) of the "phenomenon of local tissue reactivity," in 1928, numerous modifications of the original experiment were introduced in order to elicit the phenomenon in organs other than the skin. Various investigators showed that when a suitable bacterial toxin was injected into the substance of tissues, such asł the kidney, lung, intestinal wall, lymph node, joint, conjunctiva, or artery, and followed 24 hours later by an intravenous injection of toxin, the characteristic reaction of hemorrhage and necrosis appeared in the prepared tissue (2). Gratia and $\operatorname{Linz}(3)$ were the first to demonstrate that some types of tumor tissue in animals behave as though continually prepared for the Shwartzman reaction, undergoing hemorrhagic necrosis within a few hours after an intravenous injection of toxin from Gram-negative bacterial cultures.

Prior to the recognition of the Shwartzman phenomenon, Sanarelli (4) had described a set of observations which seem, in retrospect, to have involved a similar underlying mechanism. Rabbits were given an intravenous injection of live cholera organisms, and 24 hours later an intravenous injection of sterile filtrate from Bacillus coli or Bacillus proteus cultures. The majority of the animals died after the second injection, with extensive hemorrhagic lesions of the intestinal walls in which large numbers of cholera vibrios were demonstrated. Hemorrhages also occurred in the kidneys.

The experiments of Sanarelli appear to be the first demonstration of what is now termed the generalized Shwartzman phenomenon. Apitz (5), in 1934, produced the reaction by giving two intravenous injections of $B$. coli culture filtrate, 24 hours apart, and conducted a systematic study of the histopathology of the reaction. The work of Apitz, and subsequent studies by Gerber (6), Shwartzman (2), Black-Schaffer (7), and others have established that the most characteristic lesion, and the one which identifies the phenomenon as such, is bilateral cortical necrosis of the kidneys.

\footnotetext{
* This work was done under the sponsorship of the Commission on Acute Respiratory Diseases, Armed Forces Epidemiological Board, and was supported in part by the office of the Surgeon General, Department of the Army.

¥ Markle Scholar in the Medical Sciences.

The studies were also aided by grants from the National Institutes of Health, Public Health Service (HG 923), the American Heart Assaciation, The Helen Hay Whitney Foundation, and the Minnesota Heart Association.
} 
As in the local Shwartzman reaction, the generalized phenomenon is a two-stage event in which timing is an important factor. At least two intravenous injections of toxin are required, with a suitable intervening period of time. It is not possible to produce the reaction with a single injection of toxin, no matter how large the dose, except in two circumstances. The first of these is pregnancy; Apitz (8) showed that pregnant rabbits may develop bilateral renal cortical necrosis after one intravenous injection of $B$. coli endotoxin. The second is during treatment with cortisone; Thomas and Good (9) showed that cortisone-treated rabbits exhibit the generalized Shwartzman phenomenon after a single intravenous injection of meningococcal or Serratia marcescens toxin. In subsequent studies on the effects of thorotrast and trypan blue, described in an accompanying paper (10), evidence has been obtained which indicates that this effect of cortisone may be mediated through interference with detoxifying functions of the reticulo-endothelial system.

Athough there are several points of difference between the local Shwartzman phenomenon in the skin and the generalized phenomenon, which will be described later in this paper, it is reasonable to suppose that a common mechanism may be involved in both reactions. Several hypotheses have been proposed to explain the local reaction. It has been suggested that it may be due to an excessive concentration of toxin in the prepared skin site after the intravenous, or "provocative" injection, due to a supposed localizing effect of the inflammatory reaction in the skin. This explanation is contradicted by several items of evidence. First, the local injection of extremely large amounts of toxin, much larger than the amount required for intravenous provocation of the reaction, does not cause local hemorrhage in the skin. Second, repeated injections of toxin into the same skin site do not cause hemorrhage, regardless of the dosage of toxin. Third, the prepared skin site does not exhibit increased permeability to circulating dye, as is the case with other types of skin inflammation; on the contrary, the penetration of trypan blue or Evans blue into the prepared area is much less than in normal skin (11).

It was shown by Thomas and Stetson (12) that prepared skin tissues consistently exhibit a striking metabolic abnormality, which consists of the aerobic production of large amounts of lactic acid. On the basis of this observation, plus the finding that rabbits given intravenous toxin become highly susceptible to the local necrotizing effect of proteolytic enzyme injected into the skin, it was suggested that the local Shwartzman reaction might be due to an activation of tissue protease in the skin. Subsequently, Stetson (13) found that platelet-leukocyte thrombi were a conspicuous histological feature of the developing Shwartzman reaction in the skin, and suggested that occlusion of small vessels may be an initiating event in the production of hemorrhage and necrosis.

It is known that the local Shwartzman reaction can be regularly inhibited by treatment with nitrogen mustard, and the studies of Stetson and Good (14) indicate that this effect may be due to the leukopenia-producing action of nitrogen mustard. They showed that inhibition of the reaction was only demonstrable at a time approximately 72 hours after the injection of nitrogen mustard, when polymorphonuclear leukopenia occurred. When leukopenia was prevented by shielding the femoral bone marrow from the effect of nitrogen mustard the Shwartzman reaction was not inhibited. 
The present paper is the first of a series dealing with studies on the pathogenesis of the generalized Shwartzman phenomenon. In this paper a general description of the phenomenon will be presented, with an account of the conditions which are necessary for its consistent production. The effects of various agents, including nitrogen mustard, cortisone, and ACTH will be described. Certain aspects of the histopathology of the involved tissues will be considered which may have bearing on the pathogenesis of the phenomenon.

\section{Materials and Methods}

In most of the experiments to be described, meningococcal toxin was employed. This was prepared from cultures of a strain of meningococcus (44B) which was obtained through the kindness of Dr. Gregory Shwartzman. It is noteworthy that the toxin produced by this strain was much more potent than that from several freshly isolated strains of meningococci recovered in this laboratory from patients with meningitis. The following method, adapted from that described by Shwartzman (2), was employed for the preparation of toxin:-

Cultures were made by heavy seeding on the surface of Mueller-Hinton starch agar, in Kolle flasks, and grown for 48 hours in candle jars. The growth in each bottle was washed off with approximately $4 \mathrm{cc}$. of physiological saline, taking care to sweep all of the surface growth into the wash fluid. The resulting thick suspensions were centrifuged at 12,000 R.P.M. for 30 minutes, in lusteroid tubes, and the supernatant fluid stored at $4^{\circ} \mathrm{C}$. After 48 hours of storage, cultures of the fluid were negative for meningococci in all instances. In a few experiments the material was sterilized by the addition of phenol to the suspension, or by heating at $56^{\circ} \mathrm{C}$. for 30 minutes. The latter procedures did not appear to affect the potency or stability of the toxin. On several occasions it was found that batches of toxin became much diminished in activity after prolonged storage in the refrigerator at $4^{\circ} \mathrm{C}$. For this reason, new preparations were made at intervals of approximately 2 weeks, and older samples were discarded.

In a few experiments a highly purified sample of $S$. marcescens toxin obtained from Dr. Murray Shear was used. A detailed description of this material is given elsewhere in publications from Dr. Shear's laboratory (15).

Toxin was administered to all animals in a volume of $2 \mathrm{cc}$, in the ear vein. The quantity of meningococcal toxin in each experiment will be indicated by the dilution of toxin. Sterile physiological saline was used as a diluting fluid in all instances. The amount of $S$. marcescens toxin will be designated by the dry weight of the toxin; the final volume for intravenous injections of this material was also $2 \mathrm{cc}$.

Cortisone in a suspension containing $25 \mathrm{mg}$. per cc., and nitrogen mustard (methyl-bis- $\beta$ chlorethylamine hydrochloride) were supplied by Merck and Company Inc. Pituitary adrenocorticotropic hormone (ACTH) and somatotropic hormone (STH) were obtained from Armour and Company.

Young hybrid male and female albino rabbits, weighing approximately 1 kilo, were obtained from a single breeder throughout the course of the experiments. They were maintained on a diet of Purina rabbit pellets. Coccidiosis was encountered on rare occasions during the course of these experiments, and animals with this infection were not included in any of the studies to be reported.

Terminology.-Various terms have been used to describe the first and second injections of toxin which are used to produce the Shwartzman phenomenon. In the interest of clarity, the first injection will be referred to as the "preparing" dose of toxin throughout this paper. The second injection of toxin will be designated the "provoking" or "challenging" dose. The term "generalized Shwartzman reaction" will be used to apply to animals which developed 
bilateral cortical necrosis of the kidneys; the reason for restricting this term to animals with renal necrosis will be made evident.

EXPERIMENTAL

The Effect of a Single Intravenous Injection of Meningococcal Toxin

In order to determine the lethal effect of a single dose of meningococcal toxin, groups of rabbits were given intravenous injections of toxin in varying

TABLE I

The Lethal Effect of a Single Intravenous Injection of Meningococcal Toxin, and the Incidence of Bilateral Renal Cortical Necrosis and Death Following Two Intravenous Injections

\begin{tabular}{|c|c|c|c|c|}
\hline \multicolumn{2}{|c|}{ Dose of toxin } & \multirow{2}{*}{$\frac{\text { No. of rabbits }}{8}$} & \multirow{2}{*}{$\frac{\text { No. dead* }}{2}$} & \multirow{2}{*}{$\begin{array}{c}\begin{array}{c}\text { No. with bilateral } \\
\text { renal cortical } \\
\text { necrosis } \uparrow\end{array} \\
0\end{array}$} \\
\hline \multirow{6}{*}{ Single injection } & $1-2 \S$ & & & \\
\hline & $1-5$ & 8 & 1 & 0 \\
\hline & $1-10$ & 8 & 2 & 0 \\
\hline & $1-20$ & 8 & 1 & 0 \\
\hline & $1-40$ & 8 & 0 & 0 \\
\hline & $1-80$ & 8 & 0 & 0 \\
\hline \multirow{6}{*}{ Two injections } & $1-10$ & 8 & 4 & 7 \\
\hline & $1-20$ & 8 & 5 & 8 \\
\hline & $1-40$ & 8 & 3 & 7 \\
\hline & $1-80$ & 8 & 2 & 5 \\
\hline & $1-160$ & 8 & 0 & 2 \\
\hline & $1-320$ & 8 & 0 & 0 \\
\hline
\end{tabular}

* Figures refer to the number of animals dying within 24 hours after the injection of toxin. † All surviving animals were sacrificed 48 hours after the provoking injection of toxin.

$\$$ Figures refer to dilutions of meningococcal toxin. Each dose was given in volume of 2 cc.

dilutions, ranging from 1-2 to 1-80, and observed for evidences of intoxication at frequent intervals during the following 24 hours.

The animals which received the higher concentrations of toxin showed symptoms beginning between 1 and 2 hours after the injection; these consisted of weakness, rapid and labored respirations, and ruffling of the hair. The most severely ill animals remained on their sides, with arched necks, and sometimes had generalized clonic convulsions shortly before death. The mortality resulting from the various doses of meningococcal toxin is shown in Table $I$. Regardless of the dose of toxin or the severity of symptoms most of the animals recovered within 12 hours or less after injection, and remained well. All the surviving animals were sacrificed 48 hours after the injection of toxin and no gross abnormalities of any organs were seen. In a series of 265 rabbits given single injections of various doses of meningococcal toxin, during a period of 1 year, no gross lesions were demonstrable in any of the internal organs excepting in 2 animals, both of which showed mild bilateral cortical necrosis of the kidneys. This lesion was entirely similar to the type of renal necrosis occurring in the generalized 
Shwartzman phenomenon, to be described. The possibility that these two animals may have had undetected systemic infections prior to the injection of toxin cannot be excluded. In all the other rabbits given a single intravenous injection, gross and histological studies of the tissues were negative.

It was found that large doses of toxin could be given by the intradermal route without causing any symptoms of systemic intoxication. For various purposes more than 200 rabbits have been given intradermal injections of meningococcal toxin in doses of $0.5 \mathrm{cc}$. of a 1-2 dilution, and none of these animals have become ill or died.

\section{The Effect of Two Intravenous Injections of Meningococcal Toxin: the Generalized} Shwartzman Phenomenon

The response of rabbits to a second injection of meningococcal toxin was quite different from that observed after a single dose.

Many of the animals became obviously ill, usually after an interval of 4 to 6 hours. The symptoms consisted of gradually increasing weakness, unsteadiness, dyspnea, and prostration. Diarrhea occurred frequently, usually 12 hours or longer after the second injection. In most instances death occurred between 18 and 24 hours after injection. Within a few hours before death the ears of many of the animals became swollen and dark blue in color, owing to the appearance of numerous petechial hemorrhages; petechiae were not observed in other skin areas.

At autopsy, animals which died within 4 hours after the second injection of toxin showed no gross abnormalities in any internal organs. When death occurred later than 4 hours, or when the animals were sacrificed 24 hours after the second injection, bilateral cortical necrosis of the kidneys was observed in a proportion of rabbits which varied depending upon the doses of toxin administered. The relationship between dosage and the occurrence of renal lesions is illustrated in Table $I$, in which it will be seen that animals receiving two doses of meningococcal toxin in dilutions of 1-10 through 1-40 developed fatal reactions and tissue lesions more frequently than those given higher dilutions. No reactions occurred in animals receiving two doses of toxin in a dilution of $1-320$ or higher.

In general, the intensity of the kidney lesion, judged by its gross appearance, depended upon the concentration of toxin employed for the two injections.

In its mildest form, in animals receiving small doses, the lesions consisted of numerous tiny white flecks and punctate hemorrhages over the surface, and on sectioning the kidneys these white areas were seen to be confined within the cortex. Such a lesion is illustrated in Fig. 1. More severe lesions, as illustrated in Fig. 2, exhibited large, confluent, white areas of necrosis with intervening zones of hemorrhage involving the entire renal cortex. In some of the rabbits which received the larger doses of toxin, the areas of hemorrhage and necrosis extended to involve irregularly shaped areas in the medulla, and in a few animals massive hemorrhagic necrosis of the whole kidney substance was observed. An illustration of the latter lesion is shown in Fig. 3. In almost every instance, the development of bilateral cortical necrosis was accompanied by marked swelling of the kidneys. For comparison a normal kidney is shown in Fig. 4. 
Areas of hemorrhage and necrosis were usually visible in other organs in the animals which developed severe renal cortical necrosis, and in general, the more severe the renal lesion the more frequent and extensive were the lesions in other organs. The tissues involved included the lungs, heart, liver, spleen, thymus, lymph nodes, and gastrointestinal tract. Hemorrhages did not often occur in the latter tissues in the absence of renal necrosis, and many of the animals with relatively mild renal lesions showed no visible lesions in other tissues. These observations indicated that the kidney, in its gross appearance, was a more sensitive indicator of the generalized Shwartzman reaction than other tissues.

The kidney lesion appeared to be fully developed by the end of 24 hours after the second injection in all animals which survived for this length of time. In order to determine whether further progression of renal necrosis occurred after 24 hours, the following experiment was performed: Sixteen animals were given two successive injections of meningococcal toxin, diluted 1-80, spaced 24 hours apart. 8 of the animals were sacrificed 24 hours after the second injection of toxin, and the remaining 8 at 48 hours. 4 animals in each group exhibited renal cortical necrosis, and the gross appearance of the lesions did not indicate any significant difference in the severity of the lesions. For this reason, subsequent experiments dealing with the occurrence of the generalized Shwartzman reaction were regularly terminated by sacrifice of the animals 24 hours after the provoking injection of toxin.

The occurrence of bilateral cortical necrosis of the kidneys was regularly associated with a marked rise in the non-protein nitrogen content of the blood. This observation is illustrated in Table II, in which are shown the NPN levels in a group of 7 rabbits 20 hours after the provoking injection of meningococcal toxin. Four of the animals had elevations of NPN ranging from 186 to 283 , and all showed bilateral cortical necrosis of the kidneys at autopsy. In 3 rabbits the NPN was within the normal range, and the kidneys were normal. For comparison, the NPN values in 4 rabbits bled 20 hours after a single intravenous injection of toxin are included in Table II.

Histopathological Observations.-In agreement with the gross observations, the most characteristic and definitive histological lesions were regularly encountered in the kidneys. The nature and extent of the renal lesions varied depending upon the time allowed to elapse after the second injection of toxin before death or sacrifice of the animals.

The earliest alteration was the appearance of masses of eosinophilic homogeneous material within the lumen of the glomerular capillaries. This material was frequently present in the kidneys of animals sacrificed 4 hours after the second injection, at a time when there were no other renal abnormalities. In some of the glomerular capillaries the material was seen to contain a few enmeshed erythrocytes, but in no instance were more than a few platelets or leukocytes identified in the homogenous mass. Its presence as apparently free material within 
the lumen of the glomerular capillaries, and the absence of any remarkable abnormality of the capillary walls, suggest that the material is formed within the circulating blood and is not a local product or structural constituent of the vessel walls. Histochemical data, to be described elsewhere, indicate that it has the properties of a mucopolysaccharide and conforms to the standards proposed by Angevine (16) for designation as "fibrinoid" (17). It is deeply stained by the Hotchkiss-McManus modification of the Schiff reaction, as shown in a previous paper (9). It stains deep blue with azocarmine. Further studies on its nature are in progress.

In kidneys examined at a later period, between 4 and 24 hours after the provoking injection of toxin, varying degrees of tubular necrosis and hemorrhage into the parenchyma of the renal cortex were observed. Except for the occlusion of glomerular capillaries, no other vascular lesions of the kidneys were encountered. The observations suggest that the necrosis

TABLE II

The Increase in Non-Protein Nitrogen of the Blood in Rabbits with Bilateral Renal Cortical Necrosis

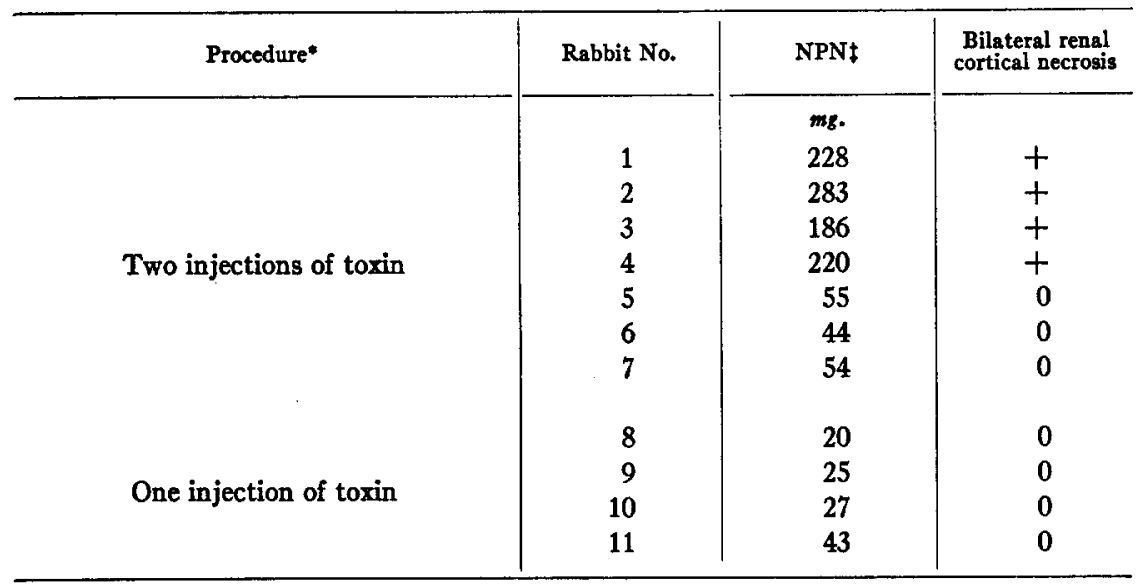

* Meningococcal toxin, 2 cc. of 1-40 dilution.

$\ddagger$ Non-protein nitrogen of blood, expressed as mg. per $100 \mathrm{cc}$.

of tubular elements in the cortex may be due primarily to interference with the glomerular capillary circulation by the deposited eosinophilic material.

Histological examination of the heart showed scattered areas of acute myofiber necrosis, with distortion, vacuolization, and basophilic staining of portions of the myofibers. The extent of myofiber necrosis varied considerably, and was not apparently related to the severity of renal lesions. Many animals with maximal degrees of renal necrosis showed little or no myocardial damage, while some with early or mild degrees of renal necrosis showed extensive myofiber necrosis. A more detailed study of the cardiac lesions will be the subject of a later report dealing with streptococcal infection and the generalized Shwartzman phenomenon.

Histological examination of other tissues, including the lungs, spleen, liver, and lymph nodes showed varying degrees of hemorrhage and necrosis involving areas of irregular sizes and shapes. A search was made for eosinophilic material within the vessels of these tissues similar to that encountered in the glomerular capillaries, with negative results.

The kidney lesion, in its gross appearance, has been accepted by most observers as the identifying lesion of the generalized Shwartzman phenomenon. 
It is easily distinguished from other spontaneous nephropathies sometimes encountered in normal stock rabbits, such as interstitial nephritis. The latter lesion is characterized by easily visible, irregularly distributed, pearl gray areas over the surface of the kidney, sometimes with pitting, without the hemorrhagic or white necrotic zones typical of bilateral cortical necrosis.

The reliability of the gross appearance of the kidney as an indicator of the generalized Shwartzman reaction depends upon the length of the time allowed to elapse between the provoking injection of toxin and death or sacrifice of the animal. It is impossible to be certain about the presence or absence of the lesion in animals which die within 12 hours or less after the provoking injection of toxin. Although some such animals show clear cut gross changes, consisting of small white flecks over the surface, others do not. In order to detect the lesion in animals during this period it is therefore necessary to depend on histological sections. On the other hand, animals which die or are sacrificed 24 hours after provocation have shown an almost complete correlation between the presence of gross and microscopic lesions and it appears that the results of experiments can be reliably evaluated on the basis of a gross inspection after this length of time

\section{The Effects of the Dosage of Toxin and the Timing of Injections on the Incidence of the Generalized Shwartzman Reaction}

One explanation for the generalized Shwartzman reaction which must be considered is that it is due to the combined and cumulative action of two doses of toxin, which if given in one injection would be rapidly lethal but when divided into two doses produce an attenuated effect, allowing progressive development of the hemorrhagic lesions which characterize the phenomenon. If this were the case, it should not be possible by two injections to produce the reaction with amounts of toxin which, when combined in one injection, have no effect.

The data presented in Table I exclude such an explanation for the phenomenon. It will be seen that a majority of animals given two doses of toxin in dilutions of $1-40$ or $1-80$ developed renal necrosis, while none of the animals receiving a single dose of toxin in dilutions of $1-2,1-5$, or $1-10$ showed any renal lesion, although most of them survived for a sufficiently long period for renal necrosis to have appeared.

The Optimal Doses of Meningococcal Toxin for Preparation and Provocation. - It is evident from the results shown in Table $I$ that the dosage of toxin was directly related to the incidence of bilateral cortical necrosis, when the same amount of toxin was used for preparation and provocation. In order to determine whether the size of either dose was of any greater importance than that of the other, an experiment was performed in which various preparing doses were tested with different provoking doses. 
Rabbits were prepared with doses of toxin in dilutions ranging from 1-10 through 1-640, and challenged 20 hours later with dilutions of 1-20,1-80, or 1-160. The results are shown in Table III. It will be seen that the highest incidence of renal necrosis occurred in the animals prepared with dilutions of 1-40 or 1-80. Paradoxically, the more concentrated doses of toxin were much less effective for preparation than higher dilutions. For example, animals given preparing dilutions of 1-10 or 1-20 exhibited a significantly lower incidence of renal necrosis than those prepared with dilutions of $1-40$ or $1-80$. On the other hand, the 1-20 dilution was more effective than smaller amounts of toxin as the provoking dose in rabbits which had been prepared with a dilution of 1-160.

From these data it appears that there is an optimal zone of toxin dosage for preparation, and excessive amounts of toxin may inhibit the reaction. No explanation is available for this finding, but it recalls the observation of

TABLE III

The Incidence of Bilateral Renal Cortical Necrosis with Various Preparing and Provoking Doses of Meningococcal Toxin

\begin{tabular}{c|c|c|c}
\hline \multirow{2}{*}{ Preparing dose of toxin } & \multicolumn{3}{|c}{ Provoking dose of toxin } \\
\cline { 2 - 4 } & $1-20^{*}$ & $1-80$ & $1-160$ \\
\hline $1-10^{*}$ & $3 / 4 \ddagger$ & $0 / 4$ & $1 / 6$ \\
$1-20$ & $5 / 5$ & $1 / 3$ & $2 / 8$ \\
$1-40$ & $5 / 5$ & $3 / 3$ & $9 / 9$ \\
$1-80$ & $4 / 4$ & $10 / 14$ & $3 / 4$ \\
$1-160$ & $4 / 4$ & $0 / 6$ & $1 / 7$ \\
$1-640$ & $0 / 4$ & $0 / 4$ & $0 / 4$ \\
\hline
\end{tabular}

* Figures refer to dilution of meningococcal toxin injected intravenously in volume of $2 \mathrm{cc}$. $\ddagger$ Numerator refers to number of rabbits with bilateral renal cortical necrosis; denominator indicates number of animals in each group.

Shwartzman (2) that the local skin Shwartzman reaction may be suppressed if excessive amounts of toxin are used for preparation of the skin.

The Optimal Time Interval between Preparation and Provocation.-In the local skin Shwartzman reaction, the optimal time between the preparing intradermal injection and the intravenous challenge ranges from 12 to 24 hours. With meningococcal toxin the interval can be lengthened to 72 or 96 hours (2), although the incidence of the reaction then diminishes. With an interval of less than 12 hours the reaction occurs irregularly and infrequently.

In order to determine the effectiveness of comparable time intervals in the generalized Shwartzman reaction, the following experiment was performed:

Rabbits were prepared by an intravenous injection of 1-40 meningococcal toxin, and groups of four each were challenged at varying times thereafter with the same dose. The animals were sacrificed 24 hours after the second injection and the incidence of renal necrosis recorded. The results are shown in Table IV. It will be seen that the optimal interval for the consistent production of renal necrosis was between 12 and 24 hours. With intervals shorter than 6 hours or longer than 4 days, the results were negative. 
The relationship between the total dosage of toxin and the incidence of the generalized Shwartzman reaction suggested the possibility that the maintenance of prolonged, more or less continual exposure to toxin might be the factor responsible, rather than the administration of two doses with a fixed time interval in between. Experiments were therefore set up to determine whether the required dose of toxin or the necessary time interval could be reduced by giving, instead of two injections of toxin, repeated injections of small amounts at hourly intervals.

TABLE IV

The Incidence of Bilateral Renal Cortical Necrosis with Various Time Intervals between the Preparing and Provoking Injections of Meningococcal Toxin

\begin{tabular}{c|c|c}
\hline \multirow{2}{*}{$\begin{array}{c}\text { Time interval between preparation } \\
\text { and provocation }\end{array}$} & \multicolumn{2}{|c}{ Generalized Shwartzman reaction $\neq$} \\
\cline { 2 - 3 } & No. dead & $\begin{array}{c}\text { No. with bilateral renal } \\
\text { cortical necrosis }\end{array}$ \\
\hline krs. & & \\
2 & 0 & 0 \\
4 & 0 & 0 \\
6 & 0 & 1 \\
8 & 0 & 1 \\
12 & 3 & 4 \\
24 & 3 & 4 \\
48 & 1 & 1 \\
72 & 0 & 1 \\
96 & 0 & 1 \\
144 & 0 & 0 \\
\hline
\end{tabular}

* 2 cc. of $1-40$ dilution of meningococcal toxin used as preparing and provoking dose.

$\$ 4$ animals in each group.

$\$$ Figures indicate number dying within 24 hours after the provoking injection. All survivors were sacrificed at 24 hours.

Two groups of rabbits were given, respectively, four and six injections of meningococcal toxin in a dilution of 1-320 every hour. 24 hours later the animals were sacrificed. None of those that received four hourly injections showed renal lesions, and only one of the group receiving six injections showed renal necrosis.

These results indicated that susceptibility to the generalized Shwartzman reaction was not enhanced by repeated exposure to toxin at short intervals.

The Effect of Cortisone and ACTH on the Lethal Action of Meningococcal Toxin

It has been suggested by various workers that the systemic action of Gramnegative bacterial toxins may be mediated through an effect on the adrenal gland, and attempts have been made by different methods to demonstrate the participation of the adrenal cortex in defense against such toxins. Lewis and 
Page (18) presented data indicating that the extreme susceptibility of adrenalectomized animals to toxin could be reversed by the administration of adrenal cortical extract, and other workers have attempted to demonstrate protection by ACTH or cortisone. Shwartzman and his coworkers $(19,20)$ stated that the local Shwartzman phenomenon could be prevented when extremely large doses of $\mathrm{ACTH}$ or cortisone were administered shortly before the provocative injection of toxin. However, Thomas and Good (9) showed that cortisone altered the response to a single injection of toxin in such a manner that intradermal toxin caused local hemorrhagic skin reactions resembling the dermal Shwartzman reaction, and a single intravenous injection resulted in bilateral cortical necrosis of the kidneys.

TABLE V

The Effects of Cortisone and ACTH on the Lethal Action of Meningococcal Toxin

\begin{tabular}{l|c|c|c|c}
\hline \multicolumn{1}{c|}{ Group } & Treatment & $\begin{array}{c}\text { No. of } \\
\text { rabbits }\end{array}$ & No. dead* & $\begin{array}{c}\text { No. with bilateral } \\
\text { renal cortical } \\
\text { necrosis }\end{array}$ \\
\hline $\begin{array}{l}\text { 1 injection of meningococcal toxin, } \\
\text { diluted 1-2 }\end{array}$ & Cortisonef & 6 & 3 & 4 \\
\hline $\begin{array}{l}\text { 2injections of meningococcal toxin, } \\
\text { diluted 1-40, 24 hours apart }\end{array}$ & Cortisone & 6 & 6 & 0 \\
\hline
\end{tabular}

* ACTH given intramuscularly in dose of $10 \mathrm{mg}$. every 6 hours, beginning 12 hours before injection of toxin and continued until end of experiment.

¥ Cortisone given intramuscularly in dose of $25 \mathrm{mg}$. daily for 3 days prior to injection of toxin.

The primary toxicity and early lethal effect of a single injection of meningococcal toxin may be due to a mechanism which differs from that involved in the Shwartzman reaction, and it seemed important to determine whether this effect could be favorably altered by cortisone. Accordingly, the following experiment was performed:-

Twelve rabbits were given an intravenous injection of $2 \mathrm{cc}$. of meningococcal toxin in a 1-2 dilution, which is an amount sufficient to cause extreme prostration and illness in all animals. 6 of the group had been pretreated for the preceding 3 days with $25 \mathrm{mg}$. of cortisone daily. The results are shown in Table V. All 12 animals became profoundly ill, and during the first 8 hours there were no discernible differences between the two groups. 1 of the 6 untreated animals died within 18 hours after the injection of toxin, and no renal or other tissue lesions were seen at autopsy. In contrast, 3 of the cortisone-treated rabbits died during this period, and 4 developed bilateral cortical necrosis of the kidneys.

This experiment demonstrates that the primary lethal effect and evidences of systemic intoxication caused by a large dose of meningococcal toxin are not 
prevented by cortisone; on the contrary, they are considerably enhanced. Similar results were obtained when the effect of cortisone or ACTH on the reaction to two intravenous injections of toxin was studied.

Three groups of 6 rabbits each were prepared and provoked by intravenous injections of 1-40 meningococcal toxin. One group was given ACTH, in a dosage of $10 \mathrm{mg}$. every 6 hours, beginning 12 hours prior to preparation. The second group received $25 \mathrm{mg}$. of cortisone daily for 3 days prior to preparation. The third group were untreated controls. The outcome is shown in Table V. The occurrence of renal necrosis and death was higher in the animals treated with ACTH or cortisone than in the controls.

The foregoing studies raised the possibility that the adrenal cortex may be involved in the mechanism of the generalized Shwartzman reaction, as a factor in the actual production of the reaction. Attempts to explore this possibility by studies in adrenalectomized animals have thus far been unsuccessful, because of the extreme toxicity of intravenously injected toxin and the difficulty in maintaining adrenalectomized rabbits alive for a sufficient time beyond the preparing injection. However, it may be noted that in experiments to be described in detail elsewhere (21), the local skin Shwartzman reaction was readily produced in rabbits after total adrenalectomy. It seems unlikely, on the basis of this indirect evidence, that the adrenal glands are involved as mediators in the Shwartzman reaction.

Pituitary somatotropic hormone (STH) has been claimed to interfere with the adverse effects of cortisone on bacterial infection (22), and the effect of this substance was therefore studied. It was found that STH, in a dose of 10 mg. per kilo daily for 3 days prior to the experiment, had no effect on the incidence of the generalized Shwartzman reaction, nor did it alter the capacity of a single injection of toxin to bring about renal necrosis in cortisone-treated rabbits.

Hematological Observations: the Role of Polymorphonuclear Leukocytes in the Generalized Shwartzman Reaction, and the Effect of Nitrogen Mustard

It has been shown by others $(2,13)$ that intravenous injections of Gramnegative bacterial toxins of the type employed for the Shwartzman reaction cause a sharp fall in the level of circulating polymorphonuclear leukocytes, which becomes evident within 30 to 60 minutes after the injection of toxin and persists for 3 to 4 hours. This is then followed by a rise in the leukocyte count to levels somewhat higher than normal. Stetson and Good (14) showed that when polymorphonuclear leukopenia was produced by the prior administration of $1.5 \mathrm{gm}$. per kilo of nitrogen mustard $\left(\mathrm{HN}_{2}\right) 3$ days earlier, the local skin Shwartzman reaction did not occur. A similar effect of $\mathrm{HN}_{2}$ on the generalized Shwartzman reaction following two intravenous injections of meningococcal toxin, or following a single injection of toxin in cortisone-treated rabbits, was observed in this laboratory (9). In the present study, the effect 
of $\mathrm{HN}_{2}$ on the generalized Shwartzman phenomenon was further investigated in the following experiments:-

First, the alterations in the level of polymorphonuclear leukocytes were determined in the course of the generalized Shwartzman reaction, produced by two intravenous injections of $S$. marcescens toxin. The results are shown in Table VI. It will be seen that the intravenous preparing injection of toxin caused a fall of polymorphonuclear leukocytes to levels of 200 or less per c. mm., beginning within 30 minutes after injection and persisting for 4 hours. After this time the leukocyte count rose to a level somewhat higher than before injection and remained elevated through the 24th hour. At this time the provoking injection of toxin was given, and the polymorphonuclear leukocytes again fell to approximately the same levels as after the first injection. 4 hours later the leukocyte count again began to rise, reaching extremely high levels by the end of the 2nd day in some animals. The development of an extremely elevated polymorphonuclear leukocyte count after the second injection of toxin was found to be consistently associated with the occurrence of bilateral renal cortical necrosis.

TABLE VI

\begin{tabular}{|c|c|c|c|c|c|c|c|c|c|c|c|c|c|}
\hline \multirow{3}{*}{$\begin{array}{c}\text { Rabbit } \\
\text { No. }\end{array}$} & \multicolumn{13}{|c|}{ Polymorphonuclear leukocytes per c.mm. of blood } \\
\hline & \multirow{2}{*}{$\begin{array}{l}\text { Before } \\
\text { toxin }\end{array}$} & \multicolumn{6}{|c|}{ Hrs. after preparation } & \multicolumn{6}{|c|}{ Hrs. after provocation } \\
\hline & & $1 / 2$ & 1 & 2 & 4 & 8 & 24 & $3 / 2$ & 1 & 2 & 4 & 8 & 24 \\
\hline 21 & 5,500 & 200 & 210 & 240 & 2,400 & 18,000 & 10,000 & 200 & 50 & 100 & 1,200 & 7,000 & 24,000 \\
\hline 22 & 3,600 & 1,800 & 800 & 100 & 800 & 4,000 & 5,600 & 01,500 & 500 & 400 & 400 & 6,500 & 20,000 \\
\hline 23 & 2,400 & 800 & 200 & 200 & 250 & 800 & 6,000 & 0200 & 100 & 100 & dead & & \\
\hline 24 & 3,200 & 400 & 200 & 200 & 100 & 1,000 & 5,000 & 01,000 & 800 & 400 & 800 & 6,000 & 16,000 \\
\hline
\end{tabular}

* Two intravenous injections of $S$. marcescens toxin, in a dose of $0.2 \mathrm{mg}$. each, were given to each rabbit. Leukocyte counts were made with blood obtained from the ear vein at the indicated times.

The effect of nitrogen mustard was determined in the following experiment:-

Rabbits were given an intravenous injection of $\mathrm{HN}_{2}$ in a dose of 1.75 per kilo. After various intervals of time, separate groups of animals were prepared by an intravenous injection of 2 cc. of a 1-40 dilution of meningococcal toxin and challenged 24 hours later with the same material. Counts of the polymorphonuclear leukocytes in the blood were made in all animals prior to the injection of $\mathrm{HN}_{2}$, and again just before the preparing injection of toxin. 24 hours after the provoking injection, all animals were sacrificed and their kidneys examined. The results are shown in Table VII.

The animals which received the preparing injection of toxin simultaneously with $\mathrm{HN}_{2}$, or 12 hours later, showed no inhibition of the generalized Shwartzman reaction. It will be noted that the polymorphonuclear leukocyte counts were within normal limits at the time of preparation in all these animals. In the group receiving the first injection of toxin 72 hours after $\mathrm{HN}_{2}$, all showed extreme leukopenia and none developed renal cortical necrosis. When the interval between the injection of $\mathrm{HN}_{2}$ and preparation with toxin was 6 or 8 days, leukopenia had disappeared and the animals were again fully susceptible to the generalized Shwartzman reaction. 
TABLE VII

Inhibition of the Generalized Shwartzman Reaction by Nitrogen Mustard; the Correlation of Inhibition with the Production of Polymorphonuclear Leukopenia by $\mathrm{HN}_{2}$

\begin{tabular}{|c|c|c|c|c|}
\hline \multirow{2}{*}{ Procedure* } & \multirow{2}{*}{ Rabbit No. } & \multicolumn{2}{|c|}{$\begin{array}{c}\text { Polymorphonuclear } \\
\text { leukocytes per } \\
\text { c. mm. of blood } \neq\end{array}$} & \multirow{2}{*}{$\begin{array}{c}\text { Bilateral } \\
\text { renal } \\
\text { cortical } \\
\text { necrosisl| }\end{array}$} \\
\hline & & $\begin{array}{l}\text { Before } \\
\mathrm{HN}_{2} \$\end{array}$ & $\begin{array}{c}\text { Before } \\
\text { preparing } \\
\text { injection } \\
\text { of toxin }\end{array}$ & \\
\hline \multirow[t]{4}{*}{ Preparation and $\mathrm{HN}_{2}$ at same time } & 1 & 4100 & - & $\mathbf{0}$ \\
\hline & 2 & 5850 & - & + \\
\hline & 3 & 3825 & - & + \\
\hline & 4 & 3500 & - & + \\
\hline \multirow{4}{*}{ Preparation 12 hours after $\mathrm{HN}_{2}$} & 5 & 3450 & 11,720 & + \\
\hline & 6 & 3120 & 7315 & + \\
\hline & 7 & 4020 & 4592 & + \\
\hline & 8 & 1470 & 2437 & + \\
\hline \multirow[t]{5}{*}{ Preparation 3 days after $\mathrm{HN}_{2}$} & 9 & 4250 & 32 & 0 \\
\hline & 10 & 1850 & 290 & 0 \\
\hline & 11 & 2090 & 325 & 0 \\
\hline & 12 & 3555 & 130 & 0 \\
\hline & 13 & 2790 & 375 & 0 \\
\hline \multirow[t]{4}{*}{ Preparation 6 days after $\mathrm{HN}_{2}$} & 14 & 3015 & 5685 & 0 \\
\hline & 15 & 3830 & 4080 & + \\
\hline & 16 & 3920 & 1577 & + \\
\hline & 17 & 6825 & 3620 & + \\
\hline \multirow[t]{5}{*}{ Preparation 8 days after $\mathrm{HN}_{2}$} & 18 & 2350 & 2750 & + \\
\hline & 19 & 2484 & 1520 & + \\
\hline & 20 & 1425 & 3275 & + \\
\hline & 21 & 1480 & 1580 & + \\
\hline & 22 & 2055 & 2020 & + \\
\hline
\end{tabular}

* All animals received $2 \mathrm{cc}$. of $1-40$ dilution of meningococcal toxin for preparation and provocation, 24 hours apart.

$\ddagger$ Leukocyte counts made with blood from ear vein shortly before the injection of $\mathrm{HN}_{2}$ or toxin.

$\$$ Nitrogen mustard in dose of 1.75 per kilo.

\| Plus sign indicates presence of renal lesion. All surviving animals sacrificed 24 hours after provocation.

It was shown by Stetson and Good (14) that clamping of the abdominal aorta at the time of injection of $\mathrm{HN}_{2}$ resulted in protection against the leukopenia-producing action of the material, presumably through shielding of the femoral marrow during the brief period in which $\mathrm{HN}_{2}$ is present in the cir- 
culating blood. Animals subjected to this procedure had normal leukocyte counts 72 hours after $\mathrm{HN}_{2}$, and were fully susceptible to the dermal Shwartzman reaction. In order to learn whether leukopenia was necessary for inhibition of the generalized Shwartzman reaction, the following experiment was performed:-

Ten rabbits were given an intravenous injection of $1.5 \mathrm{mg}$. $\mathrm{HN}_{2}$ per kilo, with a clamp applied to the abdominal aorta at the time of injection and for 5 minutes thereafter. The aorta was approached through a lower abdominal incision under nembutal anesthesia. At the same time, 9 control animals were similarly anesthetized and a clamp was applied to the aorta but released just before the injection of $\mathrm{HN}_{2} .3$ days later both groups received a preparing intravenous injection of meningococcal toxin, diluted 1-40, and 24 hours later a similar injection was given. The rabbits were sacrificed 24 hours after the provoking injection, and the kidneys examined.

7 of the 10 rabbits in which the aorta had been clamped during the injection of $\mathrm{HN}_{2}$ exhibited bilateral renal cortical necrosis. None of the animals in which the clamp was removed before the injection showed kidney lesions.

The results indicate that the generalized Shwartzman reaction, like the local skin reaction, is prevented by $\mathrm{HN}_{2}$ through an effect of the latter on the bone marrow. The association between polymorphonuclear leukopenia and protection against the generalized reaction, which implies a role of the leukocytes in the pathogenesis of bilateral renal cortical necrosis, will be discussed in a subsequent section.

\section{The Effect of Other Agents on the Generalized Shwartzman Reaction}

Materials Other Than Toxin Which Are Capable of Provoking the Local Shwartzman Reaction.-It is known that the local Shwartzman reaction, with preparation of the skin by meningococcal toxin, can be provoked by the intravenous administration of various colloidal and particulate suspensions of non-bacterial materials, such as starch, glycogen, serum, tissue extracts, kaolin, and antigen-antibody complexes $(2,12,13)$. These materials were tested for their capacity to provoke the generalized Shwartzman reaction in rabbits which had been prepared 20 hours earlier by an intravenous injection of meningococcal toxin, in the following experiments:-

Rabbits were given an intravenous injection of $2 \mathrm{cc}$. of a 1-40 dilution of meningococcal toxin, and 20 hours later groups of 4 each were given an intravenous injection of the following preparations: (a) $2 \mathrm{cc}$. of a 1 per cent kaolin, (b) $2 \mathrm{cc}$. of 5 per cent starch, (c) $10 \mathrm{cc}$. of a 10 per cent suspension of rabbit liver tissue, (d) $200 \mathrm{mg}$. of rabbit liver glycogen suspended in physiological saline, and $(c) 5$ cc. of undiluted human serum in rabbits which had been sensitized 2 weeks previously by an intravenous injection of a similar amount of serum. Each of these preparations was shown in concurrent experiments to be capable of provoking the local skin Shwartzman reaction. The results in the animals prepared with intravenous toxin were negative in all instances. None of the animals became ill and none developed renal cortical necrosis. 
Similar results were obtained in a previous study (10) with animals prepared by intravenous injections of $S$. marcescens toxin. It is evident that the provoking action of toxin is not imitated by non-bacterial materials in the generalized Shwartzman reaction, although the substances are highly active in the local Shwartzman reaction.

\section{DISCUSSION}

Bilateral cortical necrosis of the kidneys is the most constant and characteristic gross tissue lesion caused by two intravenous injections of meningococcal toxin, and is regarded as the lesion which identifies the generalized Shwartzman reaction. The incidence and intensity of this lesion, in the present experiments, were found to depend upon several factors, including the amounts of toxin employed for preparation and provocation, the time interval between the injections, and the length of time between the provoking injection and examination of the kidneys.

Areas of cortical necrosis and hemorrhage first became visible between 4 and 12 hours after provocation, and appeared to be fully developed at about 24 hours. When the same dose of toxin was used for preparation and provocation, the incidence of kidney lesions was directly related to the size of the dose. But when different amounts of toxin were used, the existence of an optimal zone of dosage became evident, with suppression of the reaction by excessively large preparing doses. No explanation is available for this finding; it may be related to the observation by Shwartzman (2) that the local skin Shwartzman reaction is inhibited when very large amounts of toxin are used for preparing the skin.

Timing is also an important factor. The highest incidence of renal necrosis occurred when the interval between preparation and provocation was 12 to 24 hours, and no reactions were produced when the interval was less than 6 or more than 96 hours.

The quality of the toxin is obviously of importance in determining the outcome. Cultures of Shwartzman's strain 44-B consistently yielded toxin which produced the reaction in 80 to 90 per cent of rabbits, while other strains of meningococci isolated in this laboratory have proved to be entirely unsatisfactory. Variable results were encountered with occasional batches of 44-B toxin which had been stored for long periods, but when fresh samples were prepared at intervals of approximately 2 weeks the results were uniform and reproducible.

The core of the problem of the Shwartzman reaction appears to be the nature and origin of the eosinophilic material which fills the glomerular capillaries. Except for its extremely homogenous appearance it might be regarded as fibrin; histological studies permit its designation as "fibrinoid." Although it occludes many of the capillaries completely, it has occasionally been seen 
lying free within the lumen and appears to be derived from the circulating blood rather than from elements of the capillary walls. The possibility that it may represent a disturbance of the coagulation mechanism is supported by the observation that heparin prevents the generalized Shwartzman reaction (23).

Occlusion of the glomerular circulation by this material could account for the necrosis of tubules in the renal cortex, since the glomerular circulation constitutes the blood supply for this tissue. A similar event involving small blood vessels in other organs may be responsible for hemorrhagic and necrotizing lesions; to settle this point will require more detailed histological studies of the involved tissues. The thrombi which Stetson (13) described in the small vessels of the skin during the local Shwartzman reaction are composed of dense aggregates of platelets and leukocytes, unlike the homogeneous material within the glomerular capillaries, but it is conceivable that cellular elements may become enmeshed in such material. It seems unlikely that the local skin reaction and the generalized Shwartzman reaction represent basically different mechanisms. Both phenomena consist of hemorrhage and necrosis, and they are similar in other important respects. The optimal time intervals between the preparing and provoking injections are comparable, the amounts of toxin required for provocation are similar, and both reactions are prevented by nitrogen mustard. The outstanding difference between the two is that the local reaction can be provoked by suspensions of colloidal or particulate substances which are completely without effect in animals prepared for the generalized reaction. No explanation for this difference is available.

The possibility that the "fibrinoid" material deposited in the glomerular capillaries may have originated in the circulating blood is of interest in the light of observations concerning the role of polymorphonuclear leukocytes in the generalized Shwartzman reaction. It has been shown that the level of circulating leukocytes is profoundly reduced as the result of intravenously injected toxin. The experiments with nitrogen mustard suggest that the presence of circulating polymorphonuclear leukocytes is necessary for the production of the generalized Shwartzman reaction. During the period when leukopenia existed following an injection of $\mathrm{HN}_{2}$, renal cortical necrosis could not be produced. In the intervals before and after the appearance of leukopenia, and when leukopenia was prevented, no inhibition by $\mathrm{HN}_{2}$ was demonstrable. The evidence suggests that an effect of toxin on polymorphonuclear leukocytes may be a principal event in the development of the Shwartzman reaction. The possibility that damage to these cells may be involved in the formation of "fibrinoid" material in the blood is under consideration.

An explanation of the generalized Shwartzman reaction would have to account for the fact that two injections of toxin must be given to produce the reaction. Evidence obtained in the present study indicates that the effect 
of two injections cannot be explained on the basis of a cumulative action, since renal necrosis can be caused by amounts of toxin which are considerably smaller than doses which have no effect when given in a single injection. A more reasonable explanation would be that the first dose of toxin impairs the capacity of the rabbit to remove or detoxify the toxin, so that the second dose is permitted to act directly on susceptible cells or tissues. Cortisone was shown to bring about a state of affairs equivalent to that produced by an intravenous injection of toxin; a single intravenous injection in cortisonetreated rabbits produced bilateral cortical necrosis of the kidneys (9). In view of the dissolution of lymphoid tissues caused by cortisone, it was suggested that the susceptibility to toxin might be due to interference with protective functions of the reticulo-endothelial system. Further evidence which supports this view is presented in the paper which follows (10).

SUMMMARY

Certain factors involved in the production of the generalized Shwartzman reaction with meningococcal toxin in rabbits were investigated. The optimal amounts of toxin for the preparing and provoking injections, and the optimal time interval between injections were determined. Under suitable conditions of dosage and timing, bilateral cortical necrosis of the kidneys was produced in a high proportion of animals. When excessive amounts of toxin were used for preparation the incidence of the reaction was reduced.

Animals undergoing the generalized Shwartzman reaction became severely prostrated within several hours after the provoking injection of toxin. The renal lesion became fully developed within 24 hours, and its occurrence was associated with a rise of the blood non-protein nitrogen. Edema and petechial hemorrhages in the ears were observed in rabbits with advanced renal lesions.

The earliest change in the kidneys in the generalized Shwartzman reaction was the appearance of homogeneous, eosinophilic material, resembling fibrinoid, within the lumen of the glomerular capillaries. Occlusion of the capillaries by this material was regarded as the cause of subsequent tubular necrosis in the renal cortex. The material appeared to be derived from the blood, rather than from the capillary walls.

Cortisone enhanced the lethal effect of a single, large dose of meningococcal toxin, as well as causing bilateral renal cortical necrosis. The generalized Shwartzman reaction produced by two injections of toxin was aggravated by cortisone and ACTH.

Profound polymorphonuclear leukopenia was produced by both the preparing and provoking injections of toxin. When leukopenia was produced before the preparing injection of toxin, by treatment with nitrogen mustard, the generalized Shwartzman reaction was inhibited. During the intervals before and after leukopenia, and when leukopenia was prevented by shielding 
the femoral bone marrow from the action of nitrogen mustard, no inhibition of the generalized Shwartzman phenomenon was demonstrable.

Various colloidal and particulate materials, which are capable of provoking the local skin Shwartzman reaction when injected intravenously, failed to provoke the generalized Shwartzman reaction.

A working hypothesis was set up to account for certain events in the generalized Shwartzman reaction.

\section{BIBLIORGAPHY}

1. Shwartzman, G., Proc. Soc. Exp. Biol. and Med., 1928, 25, 560.

2. Shwartzman, G., Phenomenon of Local Tissue Reactivity, New York, Paul Hoeber, Inc., 1937.

3. Gratia, A., and Linz, R., Compt. rend. Soc. biol., 1932, 3, 1080.

4. Sanarelli, G., Ann. Inst. Pasteur, 1924, 38, 11.

5. Apitz, K., Virchows Arch. path. Anat., 1934, 293, 1.

6. Gerber, I. E., Arch. Path., 1936, 21, 776.

7. Black-Schaffer, B., Hiebert, T. G., and Kerby, G. P., Arch. Path., 1947, 43, 28.

8. Apitz, K., J. Immunol., 1935, 29, 255.

9. Thomas, L., and Good, R. A., J. Exp. Med., 1952, 95, 409.

10. Good, R. A., and Thomas, L., J. Exp. Med., 1952, 96, 625.

11. Thomas, L., and Stetson, C. A., Jr., Proc. Soc. Exp. Biol. and Med., 1948, 69, 409.

12. Thomas, L., and Stetson, C. A., Jr., J. Exp. Med., 1949, 89, 461.

13. Stetson, C. A., Jr., J. Exp. Med., 1951, 93, 489.

14. Stetson, C. A., Jr., and Good, R. A., J. Exp. Med., 1951, 93, 49.

15. Perrault, A., and Shear, M. J., Cancer Research, 1949, 9, 626.

16. Altshuler, C. H., and Angevine, D. M., Am. J. Path., 1949, 25, 1061.

17. Kane, D. J., Good, R. A., and Thomas, L., Anat. Rec., 1952, $112,47$.

18. Lewis, L. A., and Page, I. H., J. Lab. and Clin. Med., 1946, 31, 1325.

19. Shwartzman, G., Schneierson, S. S., and Soffer, L. J., Proc. Soc. Exp. Biol. and Med., 1950, 75, 175.

20. Soffer, L. J., Shwartzman, G., Schneierson, S. S., and Gabrilove, J. L., Science, 1950, 3, 303.

21. Good, T., and Good, R. A., unpublished observations.

22. Selye, H., Canad. Med. Assn. J., 1951, 64, 477.

23. Unpublished observations. 


\section{EXPLANATION OF PLATE 29}

The photographs were made after the kidneys had been fixed in 10 per cent formalin for several days. $\times 0.9$.

Figs. 1 to 3 . The surface and cut edge of the kidneys of rabbits with the generalized Shwartzman reaction, produced by two intravenous injections of meningococcal toxin 20 hours apart. In Fig. 1, the lesion consists of numerous white areas of necrosis confined within the renal cortex. In Fig. 2, there is extensive hemorrhage throughout the cortex. In Fig. 3, the areas of necrosis and hemorrhage involve large portions of the medulla as well as the cortex.

Fig. 4. A normal rabbit kidney. 

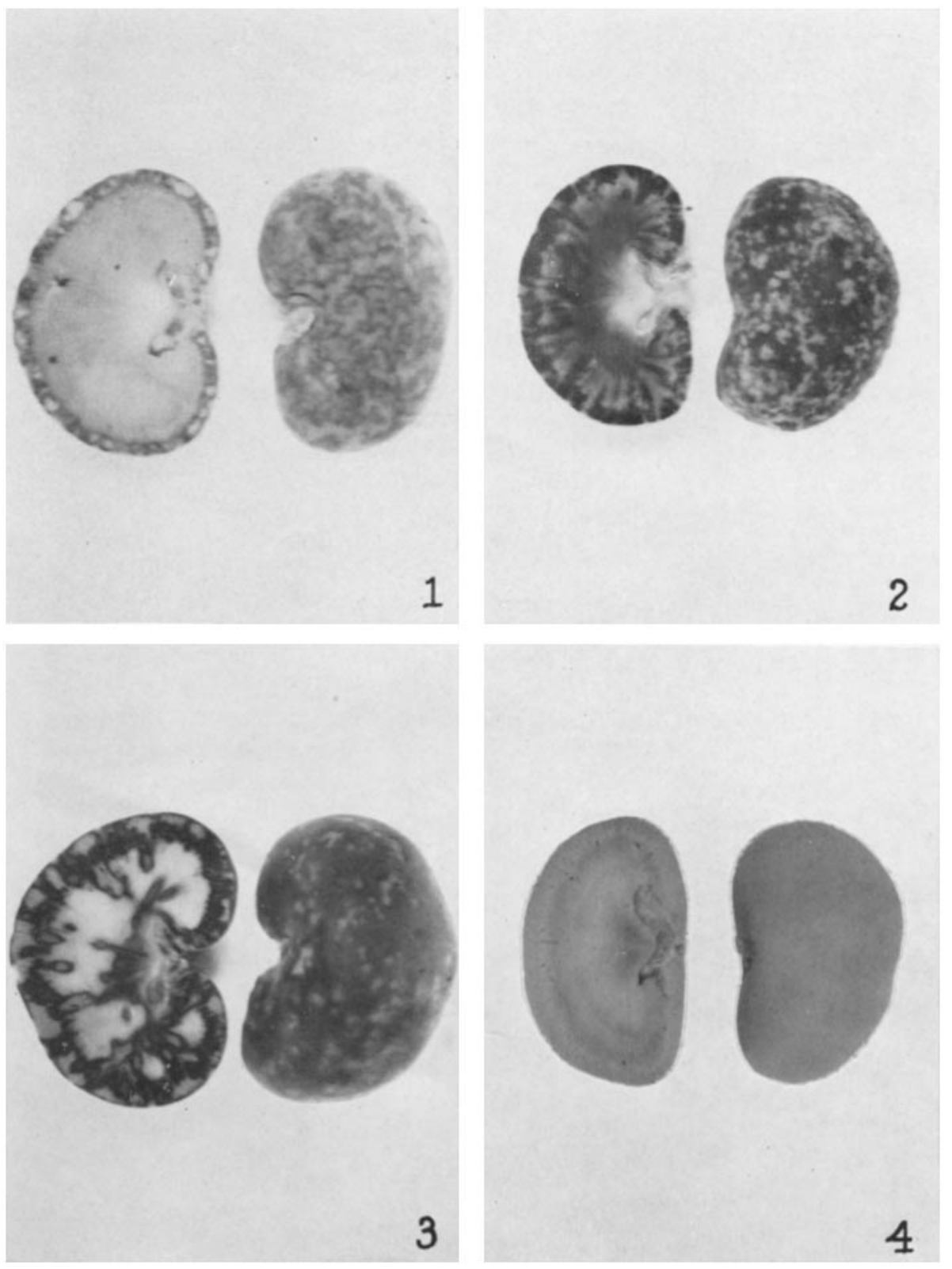

(Thomas and Good: Generalized Shwartzman reaction. I) 